\title{
Inhibition of Nitric Oxide Synthase Activity Attenuates Striatal Malonate Lesions in Rats
}

\author{
*William F. Maragos and $* \dagger$ Faye S. Silverstein \\ Departments of ${ }^{*}$ Neurology and $\dagger$ Pediatrics, University of Michigan, Ann Arbor, Michigan, U.S.A.
}

\begin{abstract}
Mitochondrial inhibitors such as malonate are potent neurotoxins in vivo. Intrastriatal injections of malonate result in neuronal damage reminiscent of "excitotoxic" lesions produced by compounds that activate NMDA receptors. Although the mechanism of cell death produced by malonate is uncertain, overactivation of NMDA receptors may be involved; pretreatment of animals with NMDA antagonists provides neuroprotection against malonate lesions. NMDA receptor activation stimulates the enzyme nitric oxide (NO) synthase (NOS). Elevated tissue levels of NO may generate highly reactive intermediates that impair mitochondrial function. We hypothesized that NO may be a mediator of malonate toxicity. We investigated whether in vivo inhibition of NO production by the NOS inhibitor $N^{\omega}$-nitro-L-arginine (NLA) would attenuate lesions produced by intrastriatal injections of malonate. We found that systemic injections of $3 \mathrm{mg} /$ $\mathrm{kg}$ of NLA significantly reduced the extent of histologic damage elicited by intrastriatal injections of $1.5 \mu \mathrm{mol}$ of malonate in adult rats. Key Words: Malonate lesions-Rat striatum-Nitric oxide-Nitric oxide synthase inhibition $-N^{\omega}$-Nitro-L-arginine. J. Neurochem. 64, 2362-2365 (1995).
\end{abstract}

Malonate reversibly inhibits the enzyme succinate dehydrogenase in both the tricarboxylic acid cycle and respiratory chain (Webb, 1966). It may also block mitochondrial anion transport (Bryla, 1980). Direct intracerebral injections of micromole quantities of malonate elicit focal necrotic lesions in adult rats. It has been speculated that lesions produced by malonate in the CNS occur via indirect overactivation of the $N$-methyl-D-aspartate (NMDA) glutamate receptor subtype (Henshaw et al., 1994). Specifically, it has been postulated that the reduction of neuronal energy stores results in neuronal depolarization, which relieves the $\mathrm{Mg}^{2+}$ gating of the NMDA-associated channel, which ultimately allows ambient levels of glutamate to activate the receptor and cause a massive influx of $\mathrm{Ca}^{2+}$ (Beal, 1992). Support for this hypothesis comes from in vivo studies in which malonate-induced lesions were attenuated by pretreatment with the noncompetitive NMDA inhibitor MK-801 (Beal et al., 1993; Greene et al., 1993).

Nitric oxide (NO) is an endogenous CNS messenger produced by the enzyme NO synthase (NOS) (Bredt and Snyder, 1990). Compounds, such as nitroprusside, that generate NO are neurotoxic when injected into the brain (Loiacono and Beart, 1992). Recent data suggest that NO is an important mediator of injury resulting from NMDA receptor overactivation (Dawson and Snyder, 1994). Inhibition of
NOS is neuroprotective in several models of cerebral ischemia and in some paradigms against excitatory amino acid toxicity (for discussion, see Dawson and Snyder, 1994); altered regulation of vascular NOS has confounded analysis of neuroprotection by NOS inhibition. The mechanism of NO toxicity is uncertain. NO reacts with superoxide free radicals to form highly reactive intermediates (Beckman et al., 1990), some of which may diminish mitochondrial oxidative phosphorylation (Bolaños et al., 1994); impaired mitochondrial function results in potentially lethal decreases in energy production. Because NMDA toxicity may, in part, be mediated by NO, we hypothesized that NO might also be a mediator of malonate toxicity. Therefore, we investigated whether in vivo inhibition of NO production by the NOS inhibitor $N^{\omega}$-nitro-L-arginine (NLA) would attenuate malonate toxicity.

\section{MATERIALS AND METHODS}

Male Sprague-Dawley rats were used in all experiments. All animal use procedures were in accordance with the NIH Guide for the Care and Use of Laboratory Animals and were approved by the University of Michigan Committee on the Use and Care of Animals. Malonate and NLA were obtained from Sigma (St. Louis, MO, U.S.A.), and $N^{\omega}$-nitro-D-arginine (NDA) was from Calbiochem (San Diego, CA, U.S.A.)

In the initial experiment, 8-week-old animals $(n=24$; weighing 190-210 g) were used. Six of them were injected intraperitoneally with $1.0 \mathrm{ml} / \mathrm{kg}$ of $0.1 M$ phosphate buffer $(\mathrm{pH} 7.4)$, and six received $3 \mathrm{mg} / \mathrm{kg}$ of NLA, i.p. One hour later, animals were anesthetized with ether and placed in a small animal stereotaxic apparatus with the incisor bar at $-2.4 \mathrm{~mm}$. Using a 30-gauge Hamilton syringe, each animal received an injection of $1.5 \mu \mathrm{mol}$ of malonate (dissolved in phosphate buffer, $\mathrm{pH} 7.4$ ) into the right striatum over $90 \mathrm{~s}$.

Resubmitted manuscript received January 16, 1995; accepted January $23,1995$.

Address correspondence and reprint requests to Dr. W. F. Maragos at Department of Neurology, University of Michigan Medical Center, Room 8301, MSRB III, Ann Arbor, MI 48109-0646, U.S.A.

Abbreviations used: NDA, $N^{\omega}$-nitro-D-arginine; NLA, $N^{\omega}$-nitroL-arginine; NMDA, $N$-methyl-D-aspartate; NO, nitric oxide; NOS, nitric oxide synthase. 
The coordinates for the striatum were AP $+0.8 \mathrm{~mm}$, ML 2.5 , relative to bregma, and DV -5.0 from the surface of the brain. Following injections, the needle was left in place for $1 \mathrm{~min}$ before the scalp was stapled shut and the animals were returned to their cages. Rectal temperatures in the control and pretreatment groups were obtained $1 \mathrm{~h}$ before, during, and 10 and $60 \mathrm{~min}$ after malonate injections. Subsequently, the experiment was replicated in 12 additional 8 -week-old animals. In two additional experiments, the identical protocol was performed using 12 -week-old rats $(n=24$; weighing 320-355 g). In each experiment, freshly prepared solutions, maintained on ice, were used.

In a third experiment, we evaluated the stereospecificity of NLA by evaluating the neuroprotective properties of its inactive isoform, NDA, in 12-week-old rats $(\mathrm{n}=12$; weighing 320-358 g). In this study, six rats were pretreated with $3 \mathrm{mg} / \mathrm{kg}$ of NDA and six with $1.0 \mathrm{ml} / \mathrm{kg}$ of $0.1 M$ phosphate buffer ( $\mathrm{pH} 7.4)$, i.p., $1 \mathrm{~h}$ before receiving a striatal injection of $1.5 \mu \mathrm{mol}$ of malonate.

Animals were killed 7 days later by decapitation, and the brains were rapidly removed and frozen on powdered dry ice. The brains were stored at $-70^{\circ} \mathrm{C}$ until sectioning. For histological analysis, $20-\mu$ m-thick frozen coronal sections obtained at $80-\mu \mathrm{m}$ intervals were thaw-mounted on gelatincoated slides. Following a 2-day exposure to paraformaldehyde vapor at room temperature, sections were stained for Nissl substance using cresyl violet. In samples from the first group of animals, adjacent $20-\mu \mathrm{m}$ sections were obtained and processed for cytochrome oxidase according to the method of Wong-Riley et al. (1978). Using computer-based image analysis (Imaging Research Inc., St. Catherines, Ontario, Canada), severity of injury was assessed by comparing the averaged regional cross-sectional area of the lesion in the 10 cresyl violet-stained sections anterior and posterior to the center of the injection site. Cytochrome oxidase-stained sections were used as a complementary method to verify extent of lesions but were not quantified. Histological comparisons were made using Mann-Whitney nonparametric analysis. The results are reported as mean \pm SEM values.

\section{RESULTS}

All of the control animals in both age groups emerged from anesthesia within $\sim 30 \mathrm{~min}$. The majority of animals pretreated with NLA exhibited 5-10 min of clonic axial extension during this period before resuming normal activity; none of the control or NDA-pretreated animals displayed this behavior. There was no difference in core temperature between the control and pretreatment groups tested at any time before, during, or up to $1 \mathrm{~h}$ following surgery (data not shown). In the initial experiment, one pretreatment animal was excluded from data analysis owing to misplacement of the needle tip. Among the 12-week-old animals, one control and one NLA-treated animals died following surgery.

In the 128 -week-old control rats that were injected with malonate, marked variability in the severity of resultant lesions was noted in both cresyl violet- and cytochrome oxidase-stained material. The average lesion area was 2.17 $\pm 0.49 \mathrm{~mm}^{2}$. Seven of 12 animals developed lesions with a mean area of $\geq 1 \mathrm{~mm}^{2}$ (range, $1.01-5.5 \mathrm{~mm}^{2}$ ); based on comparison with the mean area of the contralateral striatum over the same anterior-posterior distance, the lesions resulted in a $10-50 \%$ reduction in average area. In the remaining five animals, lesions were much less pronounced, and the cross-sectional areas ranged between 0.45 and 0.93 $\mathrm{mm}^{2}$ (4.5-9.3\% damage). Microscopic analysis of cresyl violet-stained sections of the more severe malonate-induced lesions demonstrated a necrotic core surrounded by marked neuronal loss and glial proliferation (Fig. 1A). Sections processed for cytochrome oxidase activity revealed focal reduction in reaction product coinciding with loss of Nissl stain (Fig. 1C). The smaller lesions were characterized by cell loss and gliosis extending just beyond the needle tract.

In 10 of 118 -week-old animals pretreated with NLA, the malonate-induced lesions were more homogeneous and consisted of a small circumscribed area of cell loss and gliosis immediately surrounding the needle track; there was accompanying preservation of cytochrome oxidase activity (Fig. 1B and D). The mean \pm SEM lesion area, based on average values from these 11 animals, was $0.71 \pm 0.22$ $\mathrm{mm}^{2}$ (13\% damage). Comparison of the average lesion areas between the two groups demonstrated a significant difference $(p<0.004)$; mean lesion areas in NLA-treated animals were reduced by $67 \%$ (Fig. 2 ).

The marked variability of lesion size observed in the control group of 8-week-old animals prompted us to perform the second group of experiments in older (12-week-old) rats, which were likely to be more susceptible to mitochondrial toxins (Beal et al., 1993). The lesions in the 12-week-old control animals were much more homogeneous. In this group, six of 11 animals had lesions that involved almost the entire striatum and, in one case, extended beyond it; these lesions were approximately twice as large as the most severe lesions in the younger rats and ranged from 7.6 to $9.8 \mathrm{~mm}^{2}(83-107 \%$ damage compared with the contralateral striatum). In the remaining five controls, lesions were similar in magnitude to the largest lesions in the young animals and ranged from 3.6 to $6.9 \mathrm{~mm}^{2}$ (39-75\% damage). The average area of the lesions in these 11 animals was $7.15 \pm 0.54$ $\mathrm{mm}^{2}$, significantly larger $(p<0.0001)$ than the lesions in the 8-week-old animals. In 10 of 11 of the NLA-treated animals, the average lesion area was $5.14 \pm 0.43 \mathrm{~mm}^{2}$ (range, $2.2-7.4 \mathrm{~mm}^{2}$ ); one rat had no discernible lesion and was excluded from the analysis. Compared with the intact contralateral striatum, this represents $24-80 \%$ striatal damage. In these NLA-treated animals, the average area of the malonate-induced striatal lesion was reduced $30 \%$ compared with the control $(p<0.01$; Fig. 2$)$.

In the third experiment to evaluate the effects of NDA in 12-week-old rats, striatal malonate lesions in the saline control group were homogeneous; mean lesion areas did not differ from values in the previous group of 12-week-old control animals. The average lesion area was $8.26 \pm 2.26$ $\mathrm{mm}^{2}$ (range, $6.3-10.5 \mathrm{~mm}^{2} ; 57-95 \%$ damage). In the six animals that were pretreated with NDA, the average lesion area was $8.19 \pm 1.03 \mathrm{~mm}^{2}$ (range, $6.6-9.4 \mathrm{~mm}^{2} ; 63-90 \%$ damage). There was no difference in outcome between the two groups.

\section{DISCUSSION}

We assessed the neuroprotective effects of the NOS inhibitor NLA on striatal lesions induced by the mitochondrial inhibitor malonate. Because lesions produced by malonate may be mediated, in part, by NMDA receptor activation (Henshaw et al., 1994) and because NMDA receptor activation results in the production of NO (Garthwaite et al., 1989), we speculated that impairment of NO production would attenuate malonate lesions. Although several studies have shown that NOS inhibitors are neuroprotective against 

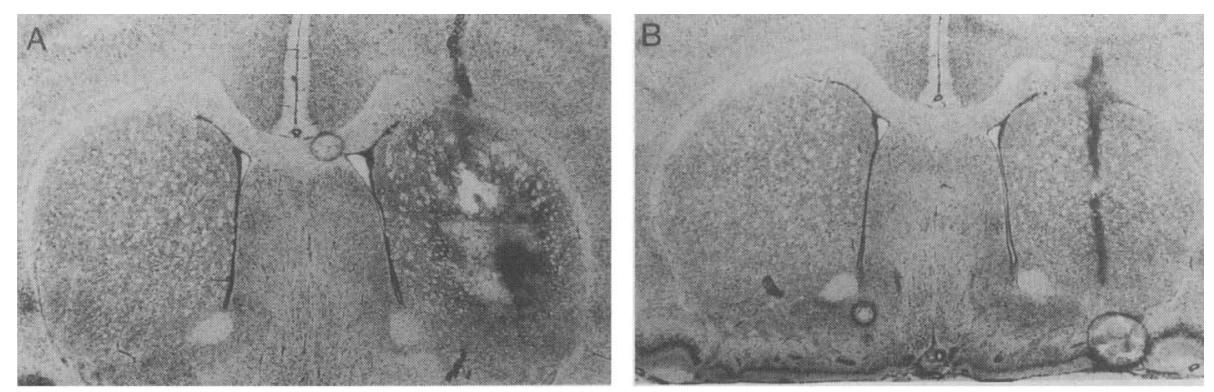

FIG. 1. Cresyl violet-stained coronal brain sections at the level of the striatum demonstrate the histopathological features of malonate lesions in 8-week-old (A) control and (B) NLA (3 mg/kg, i.p.)-pretreated animals. $A$ represents one of the larger malonate lesions, and $B$ illustrates a lesion in which the degree of neuroprotection was greater than average. Each animal
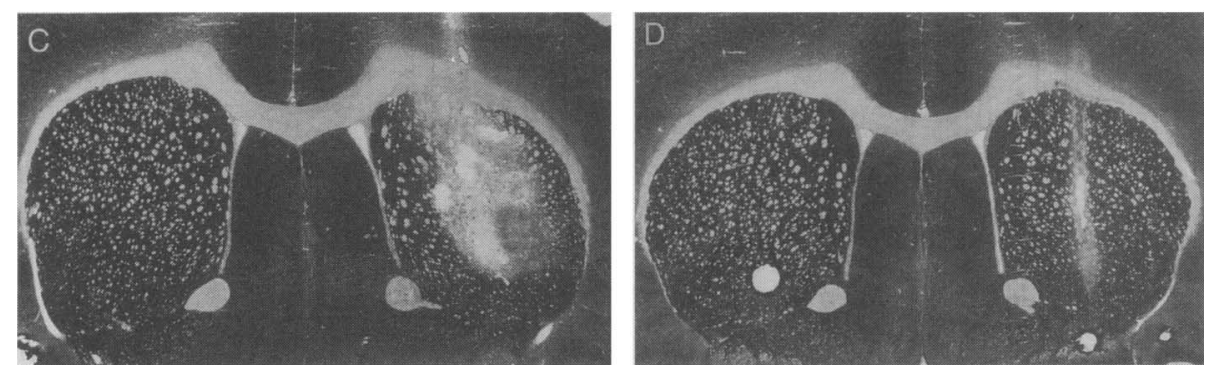
was injected with $1.5 \mu \mathrm{mol}$ of malonate and was killed 1 week later. C and D: Corresponding adjacent sections in which cytochrome oxidase activity was histochemically assayed demonstrate the extent of loss of activity closely parallels histopathological extent of tissue destruction.

NMDA receptor-mediated neurotoxicity and hypoxia/ischemia, these findings have been challenged by other reports that demonstrated either no protection or enhancement of lesions (see Dawson and Snyder, 1994). Several recent dose-response studies using NLA in different models of hypoxia/ischemia have indicated that low doses of NLA provide maximal neuroprotection (Nagafuji et al., 1993; Carreau et al., 1994); thus, we selected a pretreatment dose of $3 \mathrm{mg} / \mathrm{kg}$. Our data demonstrate that inhibition of NOS with a low dose of NLA confers significant neuroprotection against malonate-induced lesions in a stereospecific manner. Our findings are supported by a recent preliminary report demon-

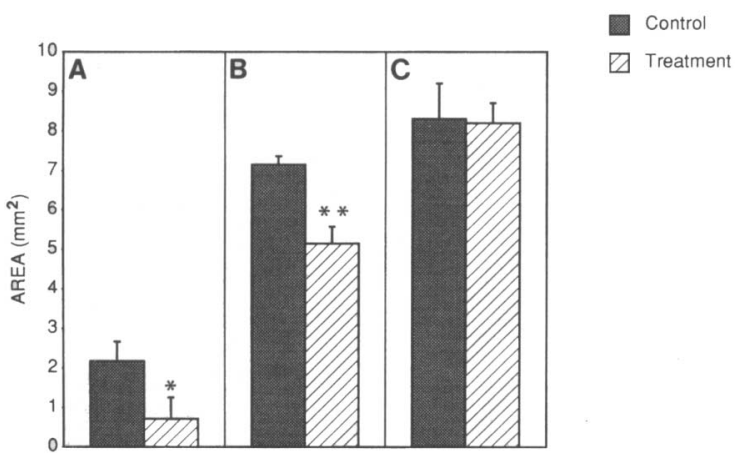

FIG. 2. Summary of the results of three independent experiments in which all of the animals received intrastriatal injections of $1.5 \mu \mathrm{mol}$ of malonate. A and $\mathbf{B}$ : Comparison of the mean areas of lesions in control and NLA-pretreated animals that were 8 (A) or 12 (B) weeks old when lesioned. In A, 12 control and 11 NLA-treated animals were compared; in B, 11 control and 10 NLA-treated animals were compared. C: Comparison of the outcome in 12-week-old animals ( $n=6$ per group) that were treated with saline or the inactive stereoisomer NDA $(3 \mathrm{mg} / \mathrm{kg})$. All animals were killed 1 week after lesioning. Lesion areas were measured in 20 coronal brain sections by computer-assisted image analysis (see Materials and Methods). ${ }^{\star} p<0.004,{ }^{\star \star} p$ $<0.01$ by Mann-Whitney test. Data are mean \pm SEM (bars) values. strating that the NOS inhibitor 7-nitroindazole also confers neuroprotection against mitochondrial toxin-induced lesions (Shulz et al., 1994).

Our data also demonstrated a marked variation in neuronal susceptibility to malonate toxicity in the younger adult rats. The extent of tissue damage determined in cresyl violetstained brain sections was confirmed using alternate sections stained for cytochrome oxidase, which demonstrated identical lesion boundaries. Beal et al. (1993) demonstrated a direct relationship between age and severity of lesions in malonate-lesioned rats; they found no reduction in neurochemical markers in the striatum of 1-month-old rats injected with $2.0 \mu \mathrm{mol}$ of malonate and a maximal reduction at 4 months. In 8-week-old young adults, the marked intergroup variability in histopathological outcome after intracerebral malonate injections was unexpected and has not been previously reported. The reason for this variability in lesion size within the group of younger animals is uncertain. These findings demonstrate that, in future studies of mitochondrial toxicity, it will be critical to consider age effects.

Our results suggest that $\mathrm{NO}$ is a mediator of malonate toxicity. The mechanisms of NO-mediated toxicity are complex. NO induces the formation of cyclic GMP (Knowles et al., 1989) and peroxynitrite (Beckman et al., 1990), each of which can have deleterious effects on cell function; increases in NO content also cause the ADP ribosylation of some proteins (Brune and Lapetina, 1989) and inhibition of mitochondrial function in astrocytes (Bolaños et al., 1994). Although these individual processes were not evaluated in this study, it is likely that more than one contribute to cell death. Additional complexity in determining the mechanism by which NLA attenuated toxicity lies in NOS itself. There are at least three forms of NOS: neuronal and endothelial (both of which are constitutive) and astrocytic (which is inducible). Because NLA is not a specific inhibitor of any single NOS isoform, it is possible that blockade of NO production by any one or all of the different enzymes contributed to the neuroprotection we observed. Availability of isozyme-specific NOS inhibitors will facilitate identifying the cellular and molecular sources of NO in malonate-lesioned brain. 
Acknowledgment: W.F.M. thanks Drs. Roger Albin and Kirk Frey for use of laboratory facilities and Timothy Desmond for his assistance. This work was supported by a grant from the Office of the Vice President for Research, University of Michigan.

\section{REFERENCES}

Beal M. F. (1992) Does impairment of energy metabolism result in excitotoxic neuronal death in neurodegenerative illnesses? Ann. Neurol. 31, 119-130

Beal M. F., Brouillet E., Jenkins B., Henshaw R., Rosen B., and Hyman B. T. (1993) Age-dependent striatal excitotoxic lesions produced by the endogenous mitochondrial inhibitor malonate. J. Neurochem. 61, 1147-1150.

Beckman J. S., Beckman T. W., Chen J., Marshall P. A., and Freeman B. A. (1990) Apparent hydroxyl radical production by peroxynitrite: implications for endothelial injury from nitric oxide and superoxide. Proc. Natl. Acad. Sci. USA 87, 1620-1624.

Bolaños J. P., Peuchen S., Heales S. J. R., Land J. M., and Clark J. B. (1994) Nitric oxide-mediated inhibition of the mitochondrial respiratory chain in cultured astrocytes. J. Neurochem. 63,910 916.

Bredt D. S. and Snyder S. H. (1990) Isolation of nitric oxide synthase, a calmodulin-requiring enzyme. Proc. Natl. Acad. Sci. USA 87, 682-685.

Brune B. and Lapetina E. (1989) Activation of a cytosolic ADPribosyltransferase by nitric oxide-generating agents. J. Biol. Chem. 264, 8455-8458.

Bryla J. (1980) Inhibitors of mitochondrial anion transport. Pharmacol. Ther. 10, 351-397.

Carreau A., Duval D., Poignet H., Scatton B., Vigé X., and Nowicki J.-P. (1994) Neuroprotective efficacy of $N^{\omega}$-nitro-L-arginine after focal cerebral ischemia in the mouse and inhibition of cortical nitric oxide synthase. Eur. J. Pharmacol. 256, 241249.
Dawson T. M. and Snyder S. H. (1994) Gases as biological messengers: nitric oxide and carbon monoxide in the brain. J. Neurosci. 14, 5147-5159.

Garthwaite J., Garthwaite G., Palmer R. M. J., and Moncada S. (1989) NMDA receptor activation induces nitric oxide synthesis from arginine in brain slices. Eur. J. Pharmacol. 172, 413416.

Greene J. G., Porter R. H. P., Eller R. V., and Greenamyre J. T. (1993) Inhibition of succinate dehydrogenase by malonic acid produces an "excitotoxic" lesion in rat striatum. J. Neurochem. 61, $1151-1154$.

Henshaw R., Jenkins B. G., Schulz J. B., Ferrante R. J., Kowall N. W., Rosen B. R., and Beal M. F. (1994) Malonate produces striatal lesions by indirect NMDA receptor activation. Brain Res. 647, 161- 166.

Knowles R. G., Palacios M., Palmer R. M. J., and Moncada S. (1989) Formation of nitric oxide from $L$-arginine in the central nervous system: a transduction mechanism for stimulation of the soluble guanylate cyclase. Proc. Natl. Acad. Sci. USA 86, $5159-5162$.

Loiacono R. E. and Beart P. M. (1992) Hippocampal lesions induced by microinjection of the nitric oxide donor nitroprusside. Eur. J. Pharmacol. 216, 331-333.

Nagafuji T., Sugiyama M., Matsui T., and Koide T. (1993) A narrow therapeutical window of a nitric oxide synthase inhibitor against transient ischemic brain injury. Eur. J. Pharmacol. 248, 325328.

Schulz J. B., Matthews R. T., Henshaw D. R., and Beal M. F. (1994) Inhibition of neuronal nitric oxide synthase (NOS) protects against neurotoxicity produced by 3-nitropropionic acid, malonate and MPTP. Soc. Neurosci. Abstr. 20, 1661.

Wong-Riley M. T. T., Merzenich M. M., and Leake P. A. (1978) Changes in endogenous enzymatic activity to DAB induced by neuronal inactivity. Brain Res. 141, 185-192.

Webb J. L. (1966) Enzyme and Metabolic Inhibitors, Vol. 2. Academic Press, New York. 